\title{
Symplectic Methods Based on the Matrix Variational Equation for Hamiltonian Systems
}

\author{
Nicoletta Del Buono, Cinzia Elia, L. Lopez \\ Dipartimento Interuniversitario di Matematica, \\ Università degli Studi di Bari \\ Via E.Orabona, 4 - I70125 Bari ITALY \\ [delbuono, elia, lopezl] @dm. uniba.it
}

\begin{abstract}
In this paper numerical methods for solving linear Hamiltonian systems are proposed. These schemes are semi-implicit procedures where no iteration is required and which are based on the numerical solution of the variational equation by means of symplectic-preserving procedures. Applications of these methods to certain nonlinear Hamiltonian systems and numerical tests are reported.
\end{abstract}

\section{Introduction}

In recent years several numerical methods have been developed to integrate systems of ODEs whose solutions preserve certain qualitative features such as orthogonality, symplecticity, isospectrality, etc (see [2], [3], [4], [6] [7]). In particular there has been a big growth of the literature on symplectic integration of Hamiltonian problems, that is models of physical systems which preserve energy (see [6],[7]). Two important features characterize Hamiltonian systems: the symplecticness of the evolution operator and the preservation of the Hamiltonian along the trajectories, hence numerical methods for such problems should inherit these properties. However in general, it is not possible to construct schemes which preserve these properties simultaneously with the same order of accuracy. Some results on symplectic methods (see [6], [7], [8]) seem to suggest that there is a better preservation of the Hamiltonian in symplectic computations over long time intervals than one expects from nonsymplectic schemes. Since, in the linear case, the solution of the associated variational equation evolves on the matrix symplectic group, here we consider semi-implicit procedures obtained by solving this variational equation by symplectic preserving methods. These procedures will be derived by projection techniques on the symplectic group or by transformation methods on the Lie algebra of the symplectic group. We also consider the nonlinear case in which such methods could be applied.

\section{Numerical methods for the non-autonomous linear case}

In this section we introduce our numerical methods in the linear case. Let us consider the Hamiltonian system

$$
u^{\prime}(t)=J A(t) u(t), \quad t>t_{0}, \quad u\left(t_{0}\right)=u_{0},
$$


with $u(t) \in \mathbb{R}^{2 n}$ and $A(t) \in \mathbb{R}^{2 n} \times \mathbb{R}^{2 n}$ symmetric matrix for each $t$ and $J$ is the canonical symplectic matrix. Let $D(t)=\frac{\partial S(t) u_{0}}{\partial u_{0}}$, that is the derivative of the evolution operator $S(t)$ with respect to the initial data $u_{0}$. It is known that $D(t)$ evolves on the symplectic group $\mathcal{S} p(2 n, \mathbb{R})=\left\{Y \in \mathbb{R}^{2 n \times 2 n} \mid Y\right.$ non singular and $\left.Y^{T} J Y=J\right\}$ and satisfies the variational equation:

$$
D^{\prime}(t)=J A(t) D(t), \quad t>t_{0}, \quad D\left(t_{0}\right)=I,
$$

(see [7], [8]). Moreover, in the linear case we have $D(t)=S(t)$, thus the solution may be given in term of the solution of the variational equation, that is

$$
u(t)=D(t) u_{0}, \quad t \geq t_{0}
$$

Then numerical methods may be derived from a computational form of the previous equation. In particular given an approximation $u_{k}$ of $u\left(t_{k}\right)$, we compute the numerical approximation $D_{k+1}$ of $D\left(t_{k+1}\right)$ by solving the variational equation

$$
D^{\prime}(t)=J A(t) D(t), \quad t \in\left[t_{k}, t_{k}+h\right], \quad D\left(t_{k}\right)=I,
$$

and then we set

$$
u_{k+1}=D_{k+1} u_{k}
$$

When $D_{k+1}$ is computed by a symplectic-preserving method, that is a method for which $D_{k+1}^{T} J D_{k+1}=J$, then (5) leads to a symplectic method for (1). In this section we consider some numerical methods which preserve during the integration the symplecticness of $D$.

\subsection{Automatic symplectic integrators}

Let us consider the $v$-stage RK method of order $p$, defined by the coefficients $\mathbf{c}=\left(c_{1}, \ldots, c_{v}\right)^{T}, \mathbf{b}=\left(b_{1}, \ldots, b_{v}\right), \mathbf{A}=\left(a_{i j}\right)$. It is well known that a Runge Kutta method applied to matrix differential system (4) is a symplectic-preserving scheme if and only if the coefficient matrix $M=\left(b_{i} a_{i j}+b_{j} a_{j i}-b_{i} b_{j}\right)$ is the zero matrix. In particular, Gauss Legendre Runge Kutta methods (GLRKv) of order $2 v$ are symplectic-preserving integrators (see [5], [6]). Thus applying a GLRK $v$ method to equation (4), we get

$$
\begin{aligned}
& D_{k+1}=I+h \sum_{i=1}^{v} b_{i} J A\left(t_{k}+c_{i} h\right) D_{k i}, \\
& D_{k i}=I+h \sum_{j=1}^{v} a_{i j} J A\left(t_{k}+c_{j} h\right) D_{k j},
\end{aligned}
$$

Hence, to find the matrix $D_{k+1}$ a linear system of size $n v$ must be solved at each step $k$ in order to obtain the internal stages $\left(D_{k 1}, \ldots, D_{k v}\right)$. To reduce the computational costs of GLRK methods one can consider symplectic-preserving schemes constructed by explicit integrators. 


\subsection{Projected methods}

In the same spirit of the projection methods on the orthogonal manifold proposed in [2], we may consider similar procedures on the symplectic manifold. A projected method consists of a two step procedure: in the first step we solve (4) by a $v$-stage explicit RK method of order $p$, that is

$$
\begin{aligned}
& D_{k+1}=I+h \sum_{i=1}^{v} b_{i} J A\left(t_{k}+c_{i} h\right) D_{k i}, \\
& D_{k i}=I+h \sum_{j=1}^{i-1} a_{i j} J A\left(t_{k}+c_{j} h\right) D_{k j}, \quad i=1, \ldots, v,
\end{aligned}
$$

and then, since $D_{k+1}$ does not belong to $\mathcal{S} p(2 n, \mathbb{R})$, it must be replaced by an approximation which is a symplectic matrix, for instance by a symplectic factorization of $D_{k+1}$.

Lemma 1. (see [1]) Let $D$ be a real $2 n \times 2 n$ matrix. Then $D$ has a factorization $D=S R$, where $S$ is unitary and symplectic, and

$$
R=\left[\begin{array}{ll}
R_{11} & R_{12} \\
R_{21} & R_{22}
\end{array}\right]
$$

with $R_{11}, R_{21}$ upper triangular matrices, and $R_{12}, R_{22} \in \mathbb{R}^{n \times n}$. Here $R_{11}$ has nonnegative diagonal entries and $R_{21}$ has a zero diagonal. Moreover, if $D$ is symplectic then $R_{21}=0$ and $R_{22}=R_{11}^{-T}$.

Given $D_{k+1}$, by using the results of the previous Lemma we compute the orthosymplectic factorization $D_{k+1}=S_{k+1} R_{k+1}$ and we set

$$
u_{k+1}=S_{k+1} u_{k}, \quad k \geq 0 .
$$

It is easy to show that: the projected method (8) is of the same order of the basic explicit RK scheme.

\subsection{Cayley methods}

A different way of devising numerical methods for symplectic flows is to take in account the matrix group structure of the set of symplectic matrices. Matrix group methods associate to the equation (4), evolving on $\mathcal{S} p(2 n, \mathbb{R})$, another ODE on the Lie algebra of the Hamiltonian matrices, that is the set

$$
\operatorname{sp}(2 n, \mathbb{R})=\left\{A \in \mathbb{R}^{n \times n} \mid A^{T} J+J A=0\right\},
$$

and solve the ODE on $\operatorname{sp}(2 n, \mathbb{R})$ by any explicit method. To return to $\mathcal{S} p(2 n, \mathbb{R})$ a suitable map is adopted. Among these methods we consider the ones based on the Cayley map for details) where the theoretical solution $D(t)$ is given in the following form:

$$
D(t)=(I-U(t))^{-1}(I+U(t)),
$$

with $U(t)$ satisfying the following ODE on the set of Hamiltonian matrices:

$$
U^{\prime}(t)=\frac{1}{2}[I-U(t)] J A(t)[I+U(t)], \quad t \in\left[t_{k}, t_{k+1}\right), \quad U\left(t_{k}\right)=0 .
$$


If the previous differential equation is solved by an explicit RK method, we get

$$
\begin{aligned}
& U_{k+1}=\frac{h}{2} \sum_{i=1}^{v} b_{i}\left(I-U_{k i}\right) J A\left(t_{k}+c_{i} h\right)\left(I+U_{k i}\right), \\
& U_{k i}=\frac{h}{2} \sum_{j=1}^{i-1} a_{i j}\left(I-U_{k j}\right) J A\left(t_{k}+c_{j} h\right)\left(I+U_{k j}\right),
\end{aligned} \quad i=1, \ldots, v,
$$

and $D_{k+1}$ may be computed by (9), that is

$$
D_{k+1}=\left(I-U_{k+1}\right)^{-1}\left(I+U_{k+1}\right) \text {. }
$$

Finally, the numerical solution of (1) may be obtained by

$$
u_{k+1}=D_{k+1} u_{k} .
$$

We will denote the group method (11)-(12) by ERKCAYp and regarding its order the following result may be derived.

Theorem 1. Let $D(t)$ be the solution of (4). Suppose that $\|D(t)\|$ is bounded by a constant $c_{1}$, where $\|\cdot\|$ is the 2 -norm on matrices. Then the method (13) based on the v-stage explicit Runge Kutta method (11) of order p, provides for $h \rightarrow 0$ the following estimate for the local truncation error

$$
\left\|u\left(t_{k+1}\right)-u_{k+1}\right\| \leq c h^{p+1}
$$

where $c$ is a constant independent on $h$.

Proof. Let $D(h)$ and $D_{1}$ be respectively the theoretical and approximated solution of (4) at $h$, and let $U(h)$ and $U_{1}$ be the theoretical and approximated solution of the associated system (10) at $h$. Since the numerical method for (10) has order $p$ we get:

$$
U(h)-U_{1}=O\left(h^{p+1}\right)
$$

with

$$
(I-U(h)) D(h)=(I+U(h)), \quad\left(I-U_{1}\right) D_{1}=\left(I+U_{1}\right),
$$

from which it follows that

$$
\begin{gathered}
(I-U(h)) D(h)-\left(I-U_{1}\right) D_{1}=U(h)-U_{1}, \\
D(h)-D_{1}-U(h) D(h)+U_{1} D_{1}+U_{1} D(h)-U_{1} D(h)=\left[U(h)-U_{1}\right], \\
\left(I-U_{1}\right)\left(D(h)-D_{1}\right)=\left(U(h)-U_{1}\right)(I+D(h)) .
\end{gathered}
$$

Hence, it follows that

$$
\begin{aligned}
\left\|D(h)-D_{1}\right\| & \leq\left\|\left(I-U_{1}\right)^{-1}\right\|\left\|U(h)-U_{1}\right\|\|I+D(h)\| \\
& \leq\left(1+c_{1}\right)\left\|\left(I-U_{1}\right)^{-1}\right\|\left\|U(h)-U_{1}\right\|
\end{aligned}
$$

that is the local error on the solution $D(h)$ is of the same order of that of $U(h)$ only if $\left\|\left(I-U_{1}\right)^{-1}\right\|$ is bounded by a constant independent by $h$, for $h \rightarrow 0$. In the case of the symplectic group, the eigenvalues of the matrix $U_{1}$ may have real part different from zero, then the above norm can not be uniformly bounded for any value of $h$, as in the case of the orthogonal group. Neverthless, $\left\|\left(I-U_{1}\right)^{-1}\right\|$ is bounded by a constant independent of $h$ for $h \rightarrow 0$. 


\section{Hamiltonian nonlinear systems}

The results of the previous sections may be applied to Hamiltonian nonlinear differential systems by using linearizing techniques. Let us consider the Hamiltonian nonlinear differential system

$$
u^{\prime}(t)=J \nabla H(u(t)), \quad t>t_{0}, \quad u\left(t_{0}\right)=u_{0},
$$

where $H \in C^{2}\left(\mathbb{R}^{2 n} \rightarrow \mathbb{R}\right)$ is the Hamiltonian function. The solution $u(t)$ may again be given as $u(t)=S(t) u_{0}$, that is in terms of evolution operator $S(t)$, but $S(t)$ now different from the Jacobian matrix function $D(t)=\frac{\partial u(t)}{\partial u_{0}}$, which satisfies the following variational equation on $\mathcal{S} p(2 n, \mathbb{R})$ :

$$
D^{\prime}(t)=J \nabla^{2} H(u(t)) D(t), \quad t>t_{0}, D\left(t_{0}\right)=I,
$$

where $\nabla^{2} H(u(t))$ denotes the Hessian matrix of $H(u)$ evaluated at the exact solution $u(t)$.

\subsection{Linearization around a stable equilibrium point}

Now, let $\tilde{u}$ a point of $\mathbb{R}^{n}$ such that $\nabla H(\tilde{u})=0$, that is $\tilde{u}$ let a stable equilibrium point for the system. Then we can consider the linearized problem:

$$
v^{\prime}(t)=J \nabla^{2} H(\tilde{u}) v(t), \quad t \in\left[t_{k}, t_{k+1}\right], \quad v\left(t_{k}\right)=u_{k},
$$

where $v(t)=u(t)-\tilde{u}$ and where $D(t)$ satisfies its variational equation

$$
D^{\prime}(t)=J \nabla^{2} H(\tilde{u}) D(t), \quad t \in\left[t_{k}, t_{k+1}\right], \quad D\left(t_{k}\right)=I .
$$

Hence we can set

$$
u_{k+1}=D_{k+1}\left(u_{k}-\tilde{u}\right)+\tilde{u},
$$

where $D_{k+1}$ is an approximation of (18) computed by a symplectic method, for instance ERKCAY.

\subsection{Transformation methods}

We suppose that our nonlinear Hamiltonian system (15) may be written in the following form

$$
u^{\prime}=A(u) u, \quad t>t_{0}, \quad u\left(t_{0}\right)=u_{0},
$$

with $A(u)$ Hamiltonian matrix for all $u \in \mathbb{R}^{2 n}$. Then, if $\tilde{u}(t)$ is an approximation of $u(t)$ on $\left[t_{k}, t_{k+1}\right]$ of order $p$, we may consider the linearized system

$$
u^{\prime}=A(\tilde{u}) u, \quad t \in\left[t_{k}, t_{k+1}\right], \quad u\left(t_{k}\right)=u_{k},
$$

the solution operator $D(t)$ of which evolves on the symplectic group. Thus we can apply to (21) the group method ERKCAY, that is, we first compute

$$
\begin{aligned}
& U_{k+1}=\frac{h}{2} \sum_{i=1}^{v} b_{i}\left(I-U_{k i}\right) A\left(u_{k i}\right)\left(I+U_{k i}\right), \\
& U_{k i}=\frac{h}{2} \sum_{j=1}^{i-1} a_{i j}\left(I-U_{k j}\right) A\left(u_{k j}\right)\left(I+U_{k j}\right), \quad i=1, \ldots, v,
\end{aligned}
$$


where $u_{k i}$ may be given by

$$
u_{k i}=\left(I-U_{k i}\right)^{-1}\left(I+U_{k i}\right) u_{k}, \quad i=1, \ldots, v,
$$

or, to avoid the inverse matrices of (23), the numerical solution $u_{k i}$ may be computed using the same explicit RK method of ERKCAY applied to (20), that is

$$
u_{k i}=u_{k}+h \sum_{j=1}^{i-1} a_{i j} A\left(u_{k j}\right) u_{k j}, \quad i=1, \ldots, v .
$$

Finally, $D_{k+1}$ and $u_{k+1}$ will be computed as in (12) and (13) respectively.

We observe that the Hamiltonian matrix $A(u)$ may be written in the following form

$$
A(u)=\left(\begin{array}{cc}
A_{11}(u) & A_{12}(u) \\
A_{21}(u) & -A_{11}^{T}(u)
\end{array}\right),
$$

where $A_{12}$ and $A_{21}$ must be symmetric matrices. Thus, since $u=(p, q)^{T}$, the condition $A(u) u=J \nabla H(u)$ is equivalent to the system

$$
A_{11}(u) p+A_{12}(u) q=-\frac{\partial H}{\partial q}, \quad A_{21}(u) p+A_{22}(u) q=\frac{\partial H}{\partial p} .
$$

Thus Hamiltonian systems for which the previous system has a solution may be transformed in the form (20). Of course, this is possible when $H(u)$ is quadratic with respect to $u$, that is $H(u)=\frac{1}{2} u^{T} S u$, where $S$ is a symmetric matrix. A different case is when $H(p, q)$ is separable, that is $H(p, q)=T(p)+V(q)$, with

$$
\frac{\partial V}{\partial q}=-A_{12}(u) q, \quad \frac{\partial T}{\partial p}=A_{21}(u) p .
$$

In this case if we assume $A_{11}=0$, and $A_{12}, A_{21}$ given by (26) we obtain a solution of $(25)$.

\section{Numerical tests}

In this section we test the proposed methods on linear and nonlinear Hamiltonian problems. All the numerical results have been obtained by Matlab codes implemented on a scalar computer Alpha 200 5/433 with 512 Mb RAM. Comparisons with methods of the same order are made in terms of symplectic error, energy error and CPU time. The symplectic error is measured by $S_{k}=\left\|D_{k}^{T} J D_{k}-J\right\|_{F}$ and the energy error by $E_{k}=\left|H\left(p_{k}, q_{k}\right)-H\left(p_{0}, q_{0}\right)\right|$, where $\|\cdot\|_{F}$ is the Frobenius norm on matrices, $p_{k}, q_{k}$ are the numerical solutions at the instant $t_{k}$ and $p_{0}, q_{0}$ are the vectors of the initial conditions. Note that we have denoted by $\operatorname{PRK} v$ the projected method of order $v$ based on the SR factorization.

Example 1. We consider the harmonic oscillator with the following separable Hamiltonian energy function $H(p, q)=T(p)+V(q)$, with $T(p)=\frac{p^{2}}{2 m}$, and 
$V(q)=\frac{k(t) q^{2}}{2}$, with $k(t)=1+10^{-4} \cos (t), m=2$ and taking as initial condition $p_{0}=q_{0}=-1$ at $t=0$. In Table 1 and 2 we show the CPU-time required, the symplectic error $S_{k}$ at the end of the integration, the maximum and the minimum value of the energy error $E_{k}$ on the integration interval $[0,100]$ for both second and fourth order methods applied with step-size $h=1$. We note that the

Table 1.

\begin{tabular}{lllll}
\hline Method & CPU-time & $S_{k}$ at 100 & $\max _{k} E_{k}$ & $\min _{k} E_{k}$ \\
\hline ERKCAY2 & 0.0667 & $2.2204 \mathrm{e}-16$ & $9.7728 \mathrm{e}-05$ & $1.0351 \mathrm{e}-06$ \\
GLRK1 & 0.0833 & $2.2204 \mathrm{e}-16$ & $1.0225 \mathrm{e}-04$ & $8.6402 \mathrm{e}-09$ \\
PRK2 & 0.1667 & 0 & 0.2640 & 0.0019 \\
\hline
\end{tabular}

behavior of the methods is very similar, but the ERKCAY2 and GLRK1 show a better conservation of the Hamiltonian than the projected methods. Moreover, ERKCAY2 requires less CPU-time.

Table 2.

\begin{tabular}{lllll}
\hline Method & CPU-time & $S_{k}$ at 100 & $\max _{k} E_{k}$ & $\min _{k} E_{k}$ \\
\hline ERKCAY4 & 0.1167 & 0 & $1.0010 \mathrm{e}-04$ & $3.2177 \mathrm{e}-07$ \\
GLRK2 & 0.1333 & $2.2204 \mathrm{e}-16$ & $9.9861 \mathrm{e}-05$ & $3.0505 \mathrm{e}-07$ \\
PRK4 & 0.1867 & 0 & 0.2520 & $7.3342 \mathrm{e}-05$ \\
\hline
\end{tabular}

\section{Example 2.}

In this example we consider the equation of the motion of a pendulum in the absence of friction:

$$
p^{\prime}(t)=-\sin q(t), \quad q^{\prime}(t)=p(t)
$$

with initial conditions $q(0)=q_{0}$ and $p(0)=p_{0}$ and Hamiltonian function

$$
H(p, q)=-\frac{1}{2} p^{2}+\cos (q)-1 .
$$

The equilibrium points of $(27)$ are $(p, q)=(0, n \pi)$ for any integer $n$. Let us consider the linearization around the stable equilibrium point $\left(p^{*}, q^{*}\right)=(0,0)$. The linearized system may be written as

$$
v^{\prime}=J\left(\begin{array}{cc}
-1 & 0 \\
0 & \cos \left(q^{*}\right)
\end{array}\right) v .
$$


Table 3.

\begin{tabular}{lll}
\hline$\left(p_{0}, q_{0}\right)$ & $\max _{k} E_{k}$ & $\min _{k} E_{k}$ \\
\hline$(1,1)$ & 0.1156 & $2.2971 \mathrm{e}-04$ \\
$\left(\frac{1}{2}, \frac{1}{2}\right)$ & 0.0077 & $1.4905 \mathrm{e}-05$ \\
$(0.2,0.1)$ & $6.6578 \mathrm{e}-05$ & $1.9315 \mathrm{e}-07$ \\
$(0,-0.4)$ & 0.0011 & $4.4668 \mathrm{e}-09$ \\
\hline
\end{tabular}

Table 3 shows the maximum and the minimum of the energy error $E_{k}$ obtained by ERKCAY2 solving the linearized systems $(28)$ on the interval $[0,100]$ with $h=0.1$ for different values of starting points. We have to observe that only if the initial points are close to the equilibrium point $(0,0)$ the solution preserves the Hamiltonian.

Fig. 1 (a) and Fig. 2 (a) plot the numerical solution of the nonlinear equation (27) with initial values $\left(p_{0}, q_{0}\right)=(0.2,0.1)$ and $\left(p_{0}, q_{0}\right)=(1,1.5)$ respectively, obtained by GLRK1 on the interval $[0,100]$ with $h=0.1$. Fig. 1 (b) and Fig. 2 (b) plot the numerical solution of the linearized system (28) obtained by ERKCAY2 starting from the same initial points with $h=0.1$. Note that only starting from initial values in a neighborhood of the equilibrium point $(0,0)$ the solution of the linearized system (28) coincides with the solution of the nonlinear problem (27).

Table 4 .

\begin{tabular}{lllll}
\hline Method & CPU-time & $S_{k}$ at 100 & $\max _{k} E_{k}$ & $\min _{k} E_{k}$ \\
\hline ERKCAY2 & 0.0833 & 0 & 1.0830 & 0.007 \\
PRK2 & 0.1833 & $2.2204 \mathrm{e}-16$ & 1.0402 & 0.0089 \\
ERKCAY4 & 0.1533 & 0 & 1.0121 & 0.0063 \\
PRK4 & 0.4000 & $2.2204 \mathrm{e}-16$ & 1.0095 & 0.0034 \\
\hline
\end{tabular}

Example 3. We now consider the nonlinear pendulum with Hamiltonian function $H(p, q)=\frac{1}{2} p^{2}+q^{2}(1-\cos q)$. Using (26) we may write the differential system in the following form

$$
\left[\begin{array}{l}
p \\
q
\end{array}\right]^{\prime}=A(p, q)\left[\begin{array}{l}
p \\
q
\end{array}\right],
$$

where

$$
A=\left(\begin{array}{c}
0-2(1-\cos q)-q \sin q \\
1
\end{array}\right) \in \operatorname{sp}(2, \mathbb{R}), \quad p, q \in \mathbb{R} .
$$

Table 4 reports the performance of second and fourth order methods on the interval $[0,10]$ with step-size $h=0.1$ and initial values $p_{0}=q_{0}=-1$ at $t=0$. 
(a)

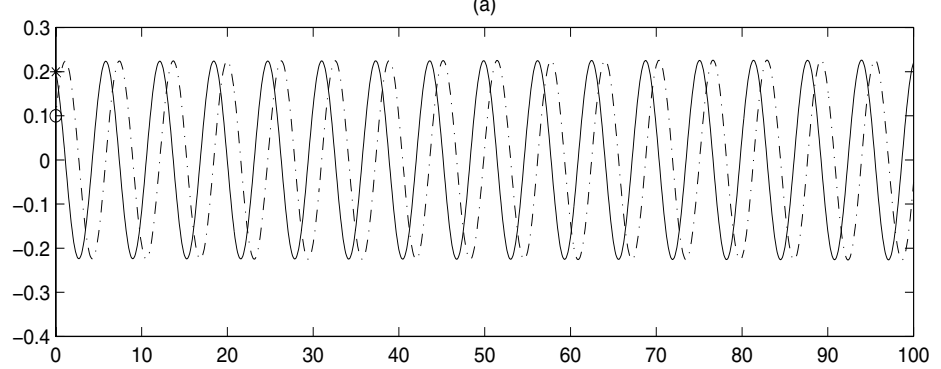

(b)

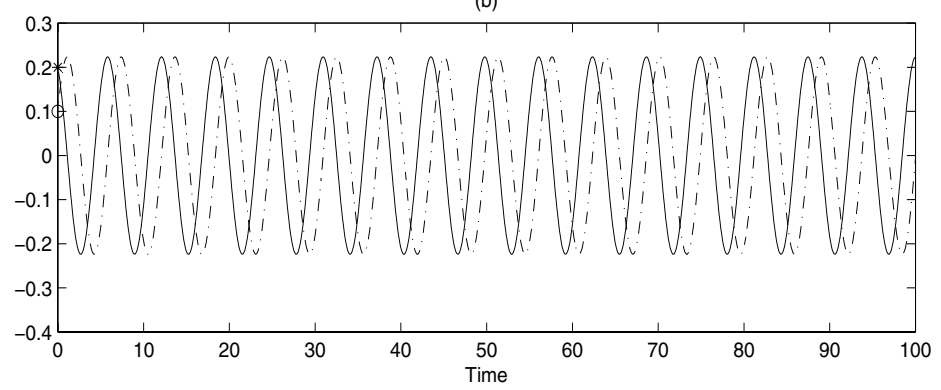

Fig. 1.

(a)

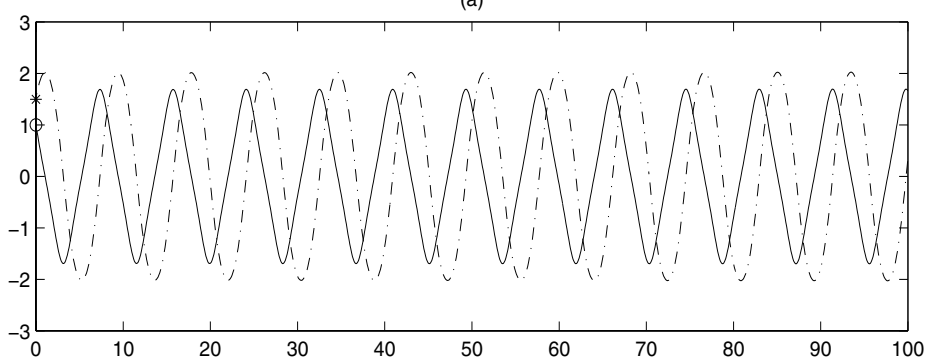

(b)

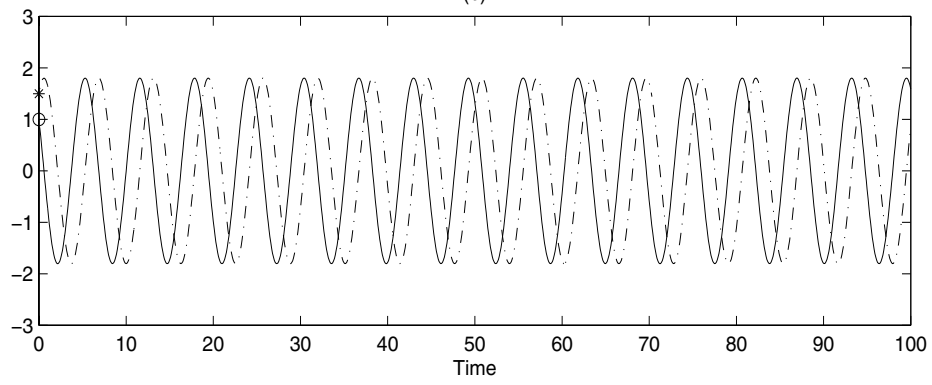

Fig. 2. 
All methods preserve the symplecticity of the matrix $D$, while the preservation of the Hamiltonian function is not satisfactory as in previous examples.

\section{References}

1. Bunse-Gerstner, A.: Matrix factorization for symplectic QR-Like methods, Linear Alg. Appl. 83 (1986) 49-77.

2. Dieci, L., Russell, D., Van Vleck, E.: Unitary integration and applications to continuous orthonormalization techniques, SIAM J. Num. Anal. 31 (1994), 261-281.

3. Diele, F., Lopez, L., Peluso, R.: The Cayley transform in the numerical solution of unitary differential systems, Advances in Comp. Math. 8 (1998). 317-334.

4. Hairer, E., Wanner, G.: Solving ordinary differential equations, Vol. II, Springer, Berlin (1991).

5. Lasagni, F.M.: Canonical Runge-Kutta methods, ZAMP 39 (1988), 952-953.

6. Sanz-Serna, J.M.: Runge Kutta schemes for Hamiltonian systems, BIT 28 (1988), 877-883.

7. Sanz-Serna, J.M., Calvo, M.P.: Numerical Hamiltonian Problems, Chapman Hall, London, (1994).

8. Stuart, A.M., Humphries, A.R.: Dynamical Systems and Numerical Analysis, Cambridge University Press (1996). 